\title{
Surrogate Reactions in the Actinide Region
}

J. T. Burke, L. A. Bernstein, N. D. Scielzo, D. L. Bleuel, S. R. Lesher, J. Escher, L. Ahle, F. S. Dietrich, R. D. Hoffman, E. B. Norman, S. A. Sheets, L. Phair, P. Fallon, R. M. Clark, J. Gibelin, C. Jewett, I. Y. Lee, A. O. Macchiavelli, M. A. McMahan, L. G. Moretto, E.

Rodriguez-Vieitez, M. Wiedeking, B. F. Lyles, C. W. Beausang, J. M. Allmond, H. Ai, J. A. Cizewski, R. Hatarik, P. D. O'Malley, T. Swan

February 29, 2008

CNR 2007 Compound Nuclear Reactions and Related Topics Fish Camp, CA, United States October 22, 2007 through October 26, 2007 
This document was prepared as an account of work sponsored by an agency of the United States government. Neither the United States government nor Lawrence Livermore National Security, LLC, nor any of their employees makes any warranty, expressed or implied, or assumes any legal liability or responsibility for the accuracy, completeness, or usefulness of any information, apparatus, product, or process disclosed, or represents that its use would not infringe privately owned rights. Reference herein to any specific commercial product, process, or service by trade name, trademark, manufacturer, or otherwise does not necessarily constitute or imply its endorsement, recommendation, or favoring by the United States government or Lawrence Livermore National Security, LLC. The views and opinions of authors expressed herein do not necessarily state or reflect those of the United States government or Lawrence Livermore National Security, LLC, and shall not be used for advertising or product endorsement purposes. 


\title{
Surrogate Reactions in the Actinide Region
}

\author{
J.T. Burke, L.A. Bernstein, N.D. Scielzo, D.L. Bleuel, S.R. Lesher, J. Escher, L. \\ Ahle, F.S. Dietrich, R.D. Hoffman, E.B. Norman, and S.A. Sheets \\ Lawrence Livermore National Laboratory, Livermore, CA 94551
}
L. Phair, P. Fallon, R.M. Clark, J. Gibelin, C. Jewett, I.Y. Lee, A.O. Macchiavelli, M.A. McMahan, L.G. Moretto, E. Rodriguez-Vieitez, M. Wiedeking

Lawrence Berkeley National Laboratory, Berkeley, California, 94720

\author{
B.F. Lyles \\ University of California, Berkeley, Berkeley, California, 94720
}

C.W. Beausang and J.M. Allmond

University of Richmond, Richmond, Virginia, 23173

H. $\mathrm{Ai}$

Yale University, New Haven, Connecticut, 06520

\author{
J.A. Cizewski, R. Hatarik, P.D. O’Malley, and T. Swan \\ Rutgers University, New Brunswick, New Jersey, 08901
}

\begin{abstract}
Over the past three years we have studied various surrogate reactions $(\mathrm{d}, \mathrm{p}),\left({ }^{3} \mathrm{He}, \mathrm{t}\right),\left(\alpha, \alpha^{\prime}\right)$ on several uranium isotopes ${ }^{234} \mathrm{U},{ }^{235} \mathrm{U},{ }^{236} \mathrm{U}$, and ${ }^{238} \mathrm{U}$. An overview of the STARS/LIBERACE surrogate research program as it pertains to the actinides is discussed. A summary of results to date will be presented along with a discussion of experimental difficulties encountered in surrogate experiments and future research directions.
\end{abstract}

Keywords: surrogate reaction, actinide, fission.

PACS: $24.87 .+\mathrm{y}, 25.40 . \mathrm{Kv}, 25.40 \mathrm{Hs}, 25.55 .-\mathrm{e}, 25.85 . \mathrm{Ge}$,

\section{INTRODUCTION}

Nuclear cross sections have many uses in basic and applied sciences. In nuclear astrophysics they help us understand the detailed evolution of stars. In nuclear reactors they guide the design and implementation of large-scale power generators. In some cases a direct measurement of a neutron induced cross section is possible. Difficulties arise when the half-life of the 
targets become short and the target becomes difficult or technically impossible to fabricate and perform a measurement. The surrogate technique circumvents these target difficulties by using a more readily available target isotope.

There are actually two different types of surrogate techniques available, the absolute and ratio method. The absolute method requires the measurement of the desired exit channel and the number of singles particles that induced the reaction [1]. In practice this can be a difficult to obtain across the entire energy region of interest due to the existence of contaminants in the target. Consider the case of measuring ${ }^{23 \mathrm{x}} \mathrm{U}\left(\alpha, \alpha^{\prime}\right)$. The uranium will form an oxide layer and then the singles spectrum of alphas becomes contaminated with scattered events from the oxygen. A solution to this is to perform a ratio experiment where the ratio of a known and unknown cross section is measured. In the case of ${ }^{237} \mathrm{U}(\mathrm{n}, \mathrm{f})$, the ratio of the number of ${ }^{238} \mathrm{U}\left(\alpha, \alpha^{\prime} f\right)$ to ${ }^{236} \mathrm{U}\left(\alpha, \alpha^{\prime} \mathrm{f}\right)$ events was multiplied by the ${ }^{235} \mathrm{U}(\mathrm{n}, \mathrm{f})$ cross section as

$$
\sigma^{23 T_{U}}\left(E_{e x}, n, f\right)=\frac{N\left[{ }^{238} U\left(E_{e x},\left(\alpha, \alpha^{\prime} f\right)\right)\right]}{N\left[{ }^{236} U\left(E_{e x},\left(\alpha, \alpha^{\prime} f\right)\right)\right]} \sigma^{235}{ }\left(E_{e x}, n, f\right) .(1)
$$

A full discussion of this technique can be found in Ref. [2].

\section{THE STARS LIBERACE APPARATUS}

The measurements for this work are performed using the Silicon Telescope Array for Reactions Studies (STARS) coupled with the Livermore BERkeley Array for Collaborative Experiments (LIBERACE). The STARS detector system consists of a segmented annular telescope that can be configured into either 48 rings and 16 sectors or 24 rings and 8 sectors. The number of telescope elements can be as many as four detectors in series to stop the most energetic particles used in a reaction. The LIBERACE detector system consists of six Ge Clover detectors arranged in a vertical plane around the main scattering chamber, as shown in Fig. 1. A full description of the experimental apparatus and electronics can be found in Ref. [3]

\section{SURROGATE REACTION EXPERIMENTS}

We have measured several fission cross sections in order to explore the applicability of the surrogate technique to the measurement of actinide fission cross sections. A brief summary of fission related experiments follows.

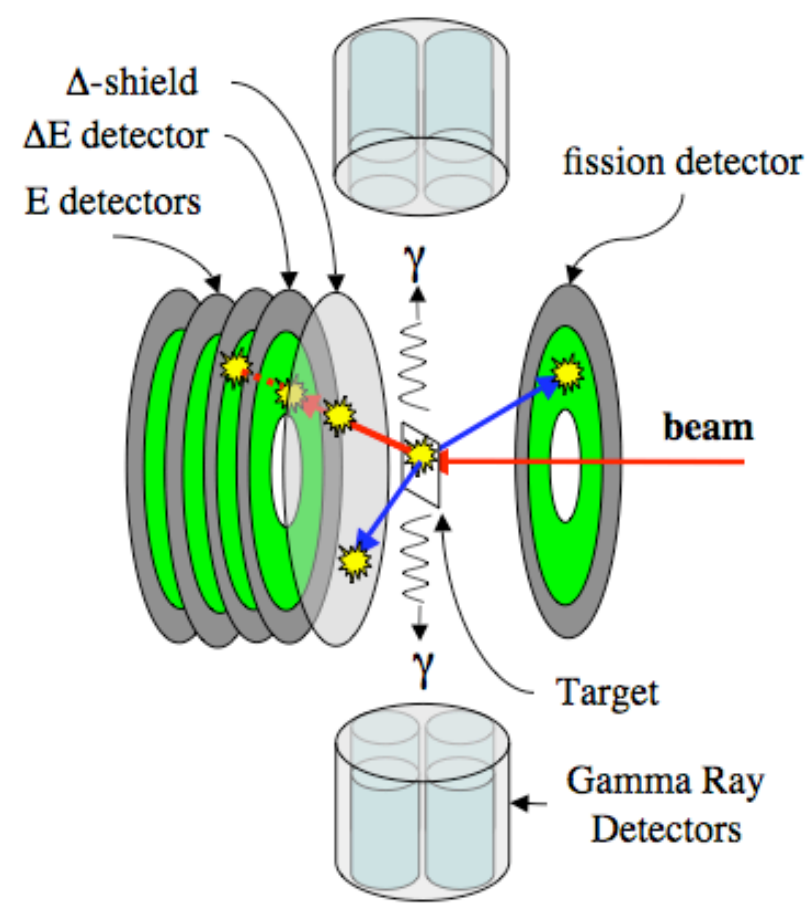

FIGURE 1. The STARS/LIBERACE apparatus is shown schematically. The silicon telescope is shown down stream of the target. The upstream fission detector is used to detect fission fragments in coincidence with outgoing scattered particles measured by the telescope. The Ge Clover detectors are shown surrounding the scattering chamber.

\section{The ${ }^{234} U\left(\alpha, \alpha^{\prime} f\right)$ and ${ }^{236} U\left(\alpha, \alpha^{\prime} f\right)$ benchmark experiment}

We have carried out benchmark experiments in order to test the surrogate ratio technique for an alpha particle entrance inelastic scattering on uranium targets. We measured the cross section ratio $\sigma\left[{ }^{234} U\left(\alpha, \alpha^{\prime} f\right)\right] / \sigma\left[{ }^{236} U\left(\alpha, \alpha^{\prime} f\right)\right]$ as a surrogate for $\sigma\left[{ }^{233} U(n, f)\right] / \sigma\left[{ }^{235} U(n, f)\right]$. The results are shown in Figure 2 below. The surrogate results are compared to the ENDF-BVII evaluation and agree well over the range from near $0 \mathrm{MeV}$ to $20 \mathrm{MeV}$ equivalent neutron energy. See Lesher [4] for a full discussion of these results. 


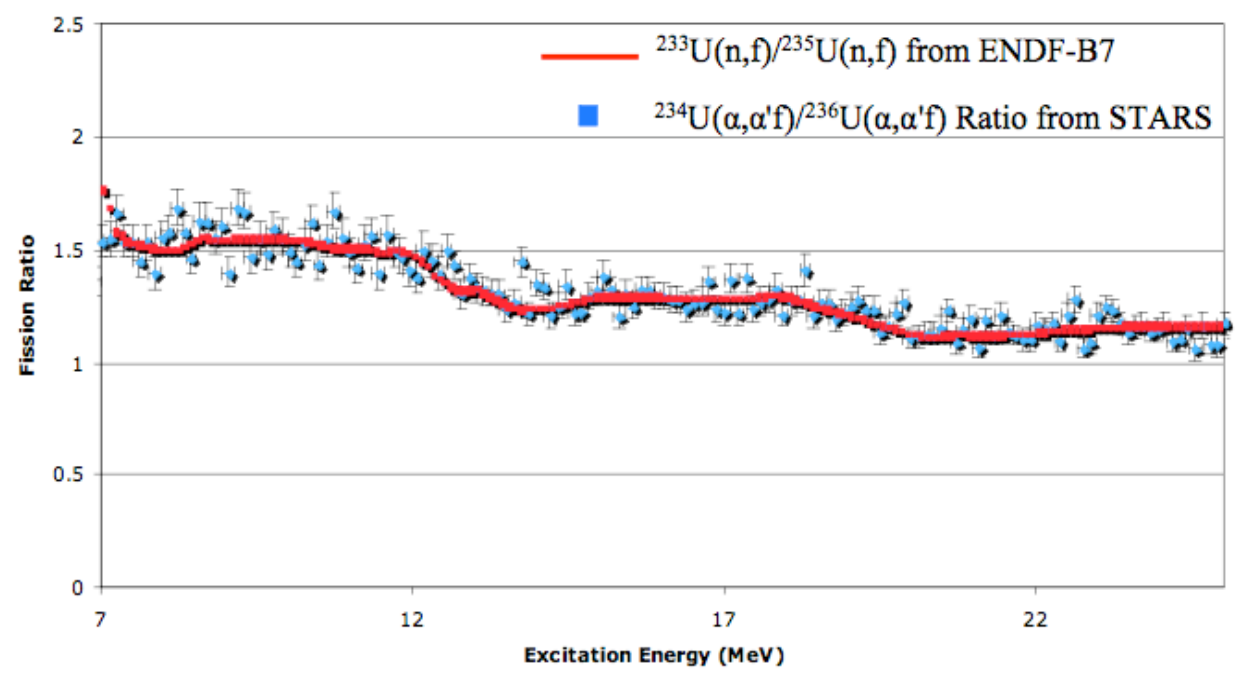

FIGURE 2. Results of the surrogate ratio of ${ }^{234} U(\alpha, \alpha$ 'f $) /{ }^{236} U(\alpha, \alpha$ 'f $)$ compared to the ENDF-BVII ratio of ${ }^{233} U(n, f) / 235 U(n, f)$. Preliminary results are shown and final results to be published by S.R. Lesher [5].

\section{The ${ }^{237} U(n, f)$ cross section from the ${ }^{238} \mathrm{U}(\alpha, \alpha$ 'f) surrogate reaction}

An application of the surrogate technique was the measurement of the ${ }^{237} U(n, f)$ cross section. The direct measurement of this cross section is extremely difficult due to the 6.75 day half-life of ${ }^{237} U$. Using the fission cross section ratio of ${ }^{238} \mathrm{U}\left(\alpha, \alpha^{\prime} f\right)$ over
${ }^{236} \mathrm{U}\left(\alpha, \alpha^{\prime} \mathrm{f}\right)$ and the known ${ }^{235} \mathrm{U}(\mathrm{n}, \mathrm{f})$ cross section, Burke et al. [3] were able to determine the ${ }^{237} \mathrm{U}(\mathrm{n}, \mathrm{f})$ cross section. The results are shown in figure 3 below. The surrogate results are compared to the ENDF-BVII and JENDL-33 evaluations. The results disagree by at least $50 \%$ at some energies. Good agreement is obtained between a more recent result from Younes et al. [6] who used a semi-empirical model and data from an early surrogate experiment.

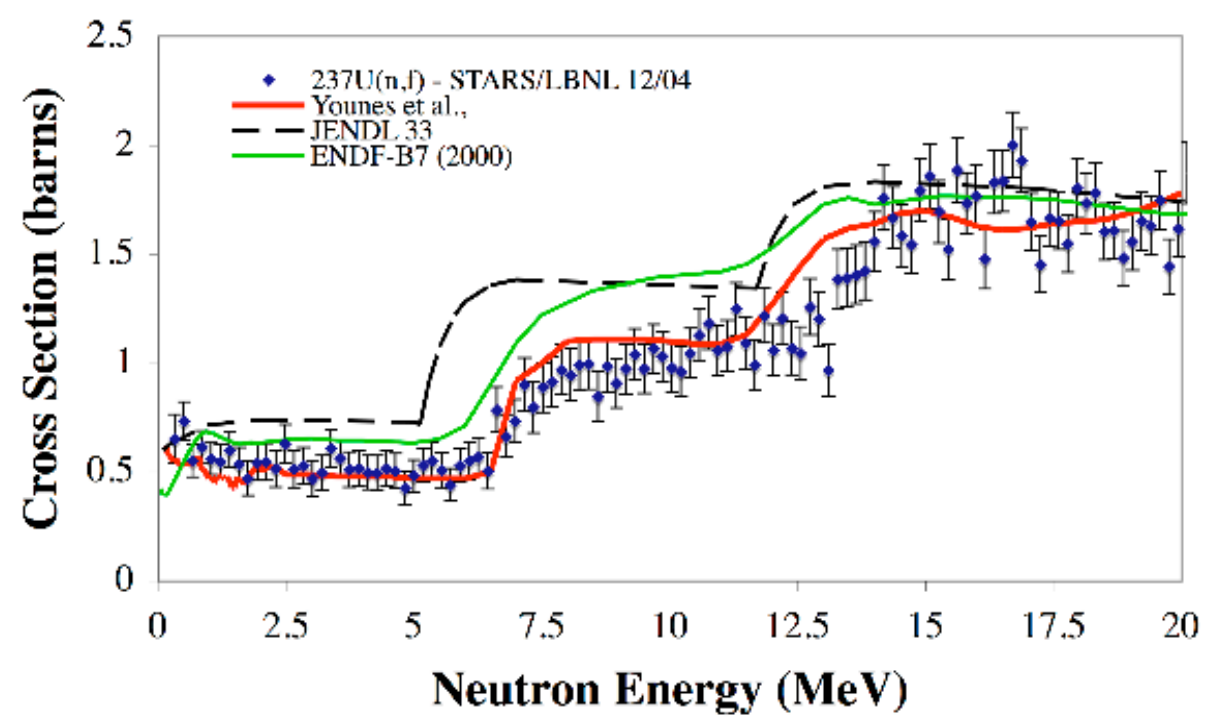

FIGURE 3. Results of the surrogate ratio of ${ }^{234} U\left(\alpha, \alpha^{\prime} f\right) /{ }^{236} U\left(\alpha, \alpha^{\prime} f\right)$ compared to the ENDF-BVII ratio of ${ }^{233} U(n, f) /{ }^{235} U(n, f)$. 


\section{The ${ }^{237} \mathrm{~Np}(\mathrm{n}, \mathrm{f})$ cross section from the ${ }^{238} \mathrm{U}\left({ }^{3} \mathrm{He}, \mathrm{tf}\right)$ surrogate reaction}

The absolute surrogate technique was used with the ${ }^{238} \mathrm{U}\left({ }^{3} \mathrm{He}, \mathrm{t}\right)$ direct reaction in the 10 to $20 \mathrm{MeV}$ equivalent neutron energy range as a surrogate for the ${ }^{237} \mathrm{~Np}(\mathrm{n}, \mathrm{f})$ reaction. The results are shown in figure 4 below. The absolute surrogate results are compared to the ENDF and JENDL evaluation and agree well over the range from $10 \mathrm{MeV}$ to $20 \mathrm{MeV}$ equivalent neutron energy. See Basunia [7] for a full discussion of these results.

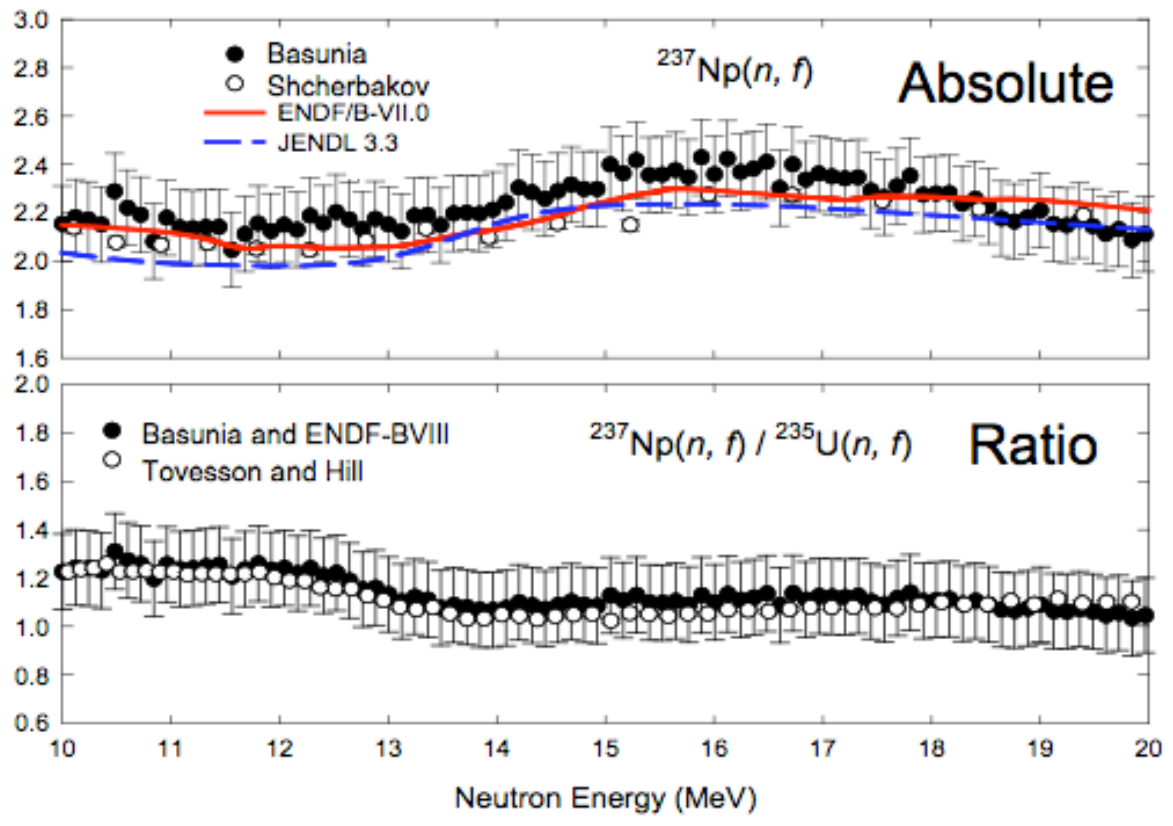

FIGURE 4. Results of the absolute surrogate ratio are compared to the ENDF, JENDL, and results of Shcherbakov [8]. The ratio of the ${ }^{237} \mathrm{~Np}(\mathrm{n}, \mathrm{f})$ to ${ }^{235} \mathrm{U}(\mathrm{n}, \mathrm{f})$ was taken in order to compare to Tovesson [9].

\section{The ${ }^{236} U(n, f)$ cross section from the ${ }^{238} \mathrm{U}\left({ }^{3} \mathrm{He}, \alpha\right.$ 'f) surrogate reaction}

The absolute and ratio surrogate technique were applied in order to extract the ${ }^{236} \mathrm{U}(\mathrm{n}, \mathrm{f})$ cross section from $\left({ }^{3} \mathrm{He}, \alpha\right)$ pickup reactions ${ }^{238} \mathrm{U}$ and ${ }^{235} \mathrm{U}[10]$. The results are shown in Fig. 5. The results of this experiment demonstrate three issues: First the surrogate ratio approach is only as good as the known cross section used to derive the unknown result. In Figure 5 one can see a large deviation between the ENDF/B-VII results and the ratio results in the 2 to 3 $\mathrm{MeV}$ region. This is arises from the rather large uncertainty/error that exists in the ${ }^{233} \mathrm{U}(\mathrm{n}, \mathrm{f})$ data in this region. Secondly, the absolute surrogate method works well provided the region is clear of contaminant species that will produce large numbers of singles events. This is apparent at approximately $7 \mathrm{MeV}$ in Figure 5 where the absolute surrogate result begins to deviate significantly from the ENDF/B-VII results. This deviation occurs exactly where bleed through from elastic $\left({ }^{3} \mathrm{He},{ }^{3} \mathrm{He}\right)$ scattering begins to contribute to the singles spectrum and continues at higher energies from a combination of ${ }^{3} \mathrm{He}$ and light nuclei $(\mathrm{O}, \mathrm{C}, \mathrm{N}))$ contaminants contributing to the singles spectrum. This is due to the imperfect particle identification in the $\Delta \mathrm{E}$ detector. Lastly, there exist spin mismatch conditions between the desired ${ }^{236} \mathrm{U}(\mathrm{n}, \mathrm{f})$ reaction and the ${ }^{238} \mathrm{U}\left({ }^{3} \mathrm{He}, \mathrm{tf}\right)$ reaction, which produce deviations from the expected results as one changes the spin of the direct reaction [10]. The effects are small (order of 5 to $10 \%$ ), but need to be corrected for with guidance from theory and modeling [2] to obtain the most precise results possible. 


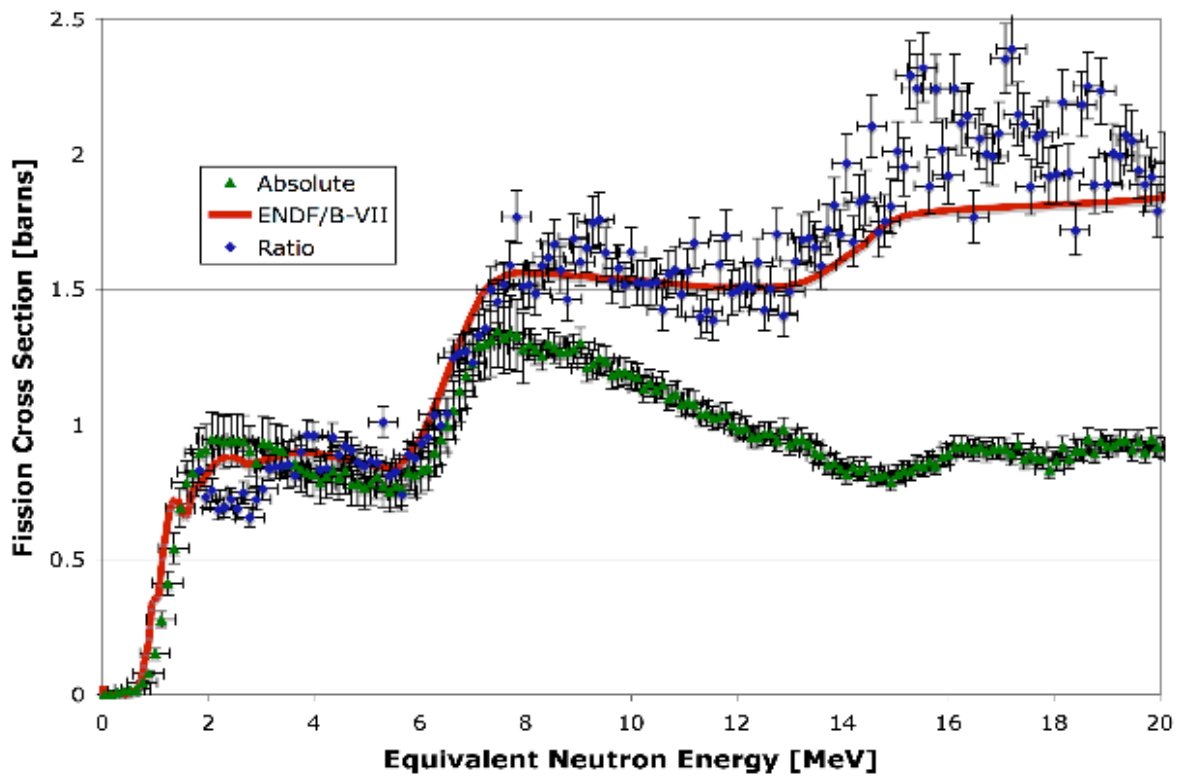

FIGURE 5. Results of the absolute surrogate ${ }^{238} \mathrm{U}\left({ }^{3} \mathrm{He}, \alpha\right.$ 'f $)$ reaction and the ${ }^{238} \mathrm{U}\left({ }^{3} \mathrm{He}, \alpha\right.$ 'f $) /{ }^{235} \mathrm{U}\left({ }^{3} \mathrm{He}, \alpha\right.$ 'f $)$ ratio compared to the ENDF/B-VII results for the desired reaction ${ }^{236} \mathrm{U}(\mathrm{n}, \mathrm{f})$ from 0 to $20 \mathrm{MeV}$.

\section{CONCLUSIONS}

We have performed several experiments examining the validity of the surrogate technique when applied to fission cross section measurements in the actinide region. In particular, we have measured the ratio of the ${ }^{234} U\left(\alpha, \alpha^{\prime} f\right)$ and ${ }^{236} U\left(\alpha, \alpha^{\prime} f\right)$ cross sections and found good agreement with the known ratio of the ${ }^{233} \mathrm{U}(\mathrm{n}, \mathrm{f})$ and ${ }^{235} \mathrm{U}(\mathrm{n}, \mathrm{f})$ cross sections, as determined from direct measurements. An absolute cross section measurement of the ${ }^{237} \mathrm{~Np}(\mathrm{n}, \mathrm{f})$ cross section has been performed using the ${ }^{238} \mathrm{U}\left({ }^{3} \mathrm{He}, \mathrm{tf}\right)$ surrogate reaction and was found to give good agreement in the measured 10 to $20 \mathrm{MeV}$ equivalent neutron energy range. We have also measured the ${ }^{237} U(n, f)$ cross section using the surrogate ratio technique and obtained good agreement with a previous effort, but disagree with previous evaluations.

We have demonstrated that the absolute and ratio surrogate technique appear to yield reliable results in the actinide region with two caveats: One must be aware that background contaminants can alter the absolute surrogate results. The ratio surrogate technique is limited by the availability of reliable cross section in the actinide region one would like to measure.

Our efforts in the future will focus on expanding this technique to $(n, \gamma)$ and $(n, 2 n)$ measurements in the actinide region.

\section{ACKNOWLEDGMENTS}

We wish to thank the 88 Inch Cyclotron operations and staff at Lawrence Berkeley National Laboratory for their support in performing these experiments over the past few years. This work was performed under the auspices of the U.S. Department of Energy by Lawrence Livermore National Laboratory in part under Contract W-7405-Eng-48 and in part under Contract DE-AC52-07NA27344 and the University of California DOE contract No. DE-AC0376SF0098 and the University of Richmond contract Nos. DE-FG05NA25929 and DE-FG02-05ER41379.

\section{REFERENCES}

1. H.C. Britt and J.B. Wilhelmy, Nucl. Sci. and Eng., 72, $222(1979)$

2. J.E. Escher and F.S. Dietrich, Phys. Rev. C, 74, 054601 (2006)

3. J.T. Burke, et al., Phys. Rev. C, 73, 054604 (2006)

4. S.R. Lesher (these proceedings)

5. S.R. Lesher et al., (to be published)

6. W. Younes and H.C. Britt, Phys. Rev. C 67, 024610 (2003).

7. M.S. Basunia et al., submitted to Phys. Rev. C

8. O. Shcherbakov et al., J. Nucl. Sci. and Tech., Supp. 2, 230, (2002)

9. F. Tovesson and T. S. Hill, Phys. Rev. C 75, 034610 (2007)

10. B.F. Lyles et al., Phys. Rev. C, 76, 014606 (2007) 